\title{
Tilted Bianchi type I model in Saez-Ballester theory of gravitation
}

\author{
D.D. Pawar ${ }^{1}$, S.P. Shahare ${ }^{2 *}$ \\ ${ }^{1}$ School of Mathematical Sciences, S.R.T.M. University, Nanded-431606, Maharashtra, India \\ ${ }^{2}$ P.R. Pote (Patil) College of Engineering and Management, Amravati-444604, Maharashtra, India
}

Available online at: www.isroset.org

Received: 22/Nov/2018, Accepted: 16/Dec/2018, Online: 31/Dec/2018

\begin{abstract}
Present paper deals with the study of an anisotropic tilted Bianchi type-I cosmological model in Kasner form filled with non-viscous fluid in the framework of Saez-Ballester theory of gravitation. Exact solutions of field equations are obtained using a special condition. The physical parameters of the model are obtained and discussed $\mathrm{s}$ which yields a constant positive value of deceleration parameter.
\end{abstract}

Keywords - Tilted cosmological model, Kasner metric, Saez-Ballester theory, deceleration parameter.

\section{INTRODUCTION}

In recent years, there has been a considerable interest in investigating Bianchi cosmologies which play important role in theoretical cosmology presenting spatially homogeneous universe, have been much studied since 1960s. Various aspects of the physical universe can be investigated with the help of homogeneous cosmological models. In this work we focused our attention on Tilted Bianchi type-I model in Kasner form. Spatially homogeneous and anisotropic cosmological models in which the matter does not move orthogonal to the hyper surface of homogeneity. Such type of models are called tilted cosmological models. Homogeneous and anisotropic cosmological models have been studied widely in the framework of general relativity. These models are more restricted than that of the inhomogeneous models. The general dynamics of tilted cosmological models have been studied by King and Ellis [1], Ellis and King [2]. According to Collins and Ellis [3], a tilted model is spatially homogeneous relative to observers whose world line are orthogonal relative to the group orbits but it is spatially inhomogeneous relative to observers commoving with the fluid. In a tilted Bianchi cosmology the tilt can become extreme in a finite time as measured along the fluid congruence, with the result that the group orbits become time-like. This means that the models are no longer spatially homogeneous. Different aspects of tilted cosmological models have been developed and discussed by some authors such as Matravens et al. [4], Dunn et al.[5], Horwood et al. [6], Hewit et al. [7], Aposotolopoulos [8] . Bali and Sharma [9], Pradhan and Rai [10] studied tilted Bianchi type-I models. Tilted plane symmetric cosmological models with heat conduction and disordered radiation have studied by Pawar et al. [11]. Bali and Meena [12] have obtained tilted cosmological models filled with disordered radiation of perfect fluid and heat flow. Pawar and Dagwal [13] have discussed confirmally flat tilted cosmological models. Sahu [14-15] obtained tilted Bianchi type- $\mathrm{VI}_{0}$ and Bianchi typeIII cosmological models in Saez - Ballester theory of gravitation. Pawar and Dagwal [16-17] have discussed tilted Kantowski-Sachs cosmological models with disordered radiation in Saez - Ballester theory and tilted Kasner-type cosmological model in Brans-Dicke theory.

In present work we studied a scalar- tensor theory named Saez-Ballester theory of gravitation which has been developed by Saez and Ballester [18], in which the metric is coupled with a dimensionless scalar field. This is the modified theory of the Einstein's theory of relativity. Shri Ram and Tiwari [19], Singh and Shri Ram [20] studied several aspects of Saez - Ballester theory. Katore [21-22] obtained exact solutions of five dimensional FRW metric in the presence of perfect fluid and that of Kantowski-Sachs Dark Energy cosmological model using a scalar tensor theory of gravitation proposed by $\mathrm{Saez}$ and Ballester. Bianchi-VI ${ }_{1}$ space time with wet dark fluid in Saez-Ballester theory has obtained by Mishra and Sahoo [23]. Also Pawar et al.[24-25] studied Marder's Dark Energy Model in Saez Ballester theory. Recently Verma et al. [26] investigated Hypersurface-homogeneous cosmological models with anisotropic dark energy in Saez-Ballester theory of gravitation.

In Bianchi type -I metric of Kasner form, the cosmological scale factors evolve as a power law in time. The Kasner space times are spatially homogeneous and anisotropic. It is the special class of Bianchi type-I space time which has been helped to study the properties of anisotropically expanding cosmologies as well as it is used to describe the early stages of evolution of the universe. Dynamics of the anisotropy is one of the powerful tool for analyzing anisotropic expansion in three dimensional. 
According to Brevik et.al. [27-28], Kasner metric with viscous fluid in Einstein's theory is not anisotropic and that of Kasner metric with non-viscous fluid satisfying Zel'dovich equation of state is to be anisotropic. The Bianchi type-I metric of Kasner form in general relativity filled with viscous fluid satisfying the dominant energy condition (DEC) [29] along with second law of thermodynamics has been examined by Cataldo et. al. [30]. In that work it was shown that the Kasner form do not permit the universe to be anisotropic. Cataldo et al [31] studied Kasner model filled with adiabatic anisotropic matter in the framework of the holographic principle. Halpern [32] discussed the behavior of Bianchi type- I model in Kasner form with Induced Matter. Cataldo et al [33] proved that- Kasner form permits the universe to be anisotropic in scalar tensor theories. While according to Lee [34], for a more general Bianchi type-I metric with a perfect fluid it is possible in the Brans-Dicke theory of gravity.

In the present work, we studied Tilted Bianchi type-I Cosmological model of Kasner form in the scalar tensor theory of gravitation proposed by Saez and Ballester [18]. This paper is outlined as follows: Section I contains the introduction of previous work, Section II presents the brief introduction of the field equations in Saez - Ballester theory of gravity. Section III determines some physical parameters of the model, section IV describes results and discussion, section V summarizes our conclusions and some final remarks. Lastly in section VI, references are listed.

\section{METRIC AND FIELD EQUATIONS}

We consider the Bianchi type-I space time of Kasner form

$d t^{2}=-d t^{2}+t^{2 \xi_{1}} d x^{2}+t^{2 \xi_{2}} d y^{2}+t^{2 \xi_{3}} d z^{2}$,

where $\xi_{1}, \xi_{2}$ and $\xi_{3}$ are Kasner constant parameters.

The corresponding Ricci Scalar

$$
\begin{gathered}
R=2\left(\xi_{1}+\xi_{2}+\xi_{3}\right)-\left(\xi_{1}+\xi_{2}+\xi_{3}\right)^{2} t^{-2}-\left(\xi_{1}{ }^{2}+\xi_{2}{ }^{2}\right. \\
\left.+\xi_{3}{ }^{2}\right) t^{-2}
\end{gathered}
$$

The Einstein's field equations in Saez-Ballester for the combined scalar and tensor field are:

$G_{i}^{j}-\omega \varphi^{n}\left(\varphi_{, i} \varphi^{j}-\frac{1}{2} g_{i}^{j} \varphi_{, a} \varphi\right)=T_{i}^{j}$

where $\mathrm{n}$ is an arbitrary constant, $\omega$ is a dimensionless coupling constant .

And the scalar field $\varphi$ satisfies the equation

$$
2 \varphi^{n} \varphi_{; i}^{j}+n \varphi^{n-1} \varphi_{, a} \varphi^{, a}=0
$$

Here comma and semicolon denote partial and covariant differentiation respectively.
The energy momentum tensor for the perfect fluid with heat conduction vector given by Ellis [2] is

$T_{i}^{j}=(p+\rho) u_{i} u^{j}+p g_{i}^{j}+q_{i} u^{j}+q_{j} u^{i}$

together with $g_{i j} u^{j} u^{j}=-1$

$$
q_{i} q^{j}>0 \text { and } q_{i} u^{i}=0
$$

Where $p$ is the pressure, $\rho$ is the energy density, $q^{i}$ is the heat conduction vector orthogonal to the fluid flow vector $u^{i}$. The fluid flow vector $u^{i}$ has the components $\left(\frac{\sinh \alpha}{t \xi_{1}}, 0,0, \cosh \alpha\right)$ satisfying equation (5) and $\alpha$ is the tilt angle. From equation (4), we have

$T_{1}^{1}=(p+\rho) \sinh ^{2} \alpha+p+2 q_{1} \frac{\sinh \alpha}{t^{\xi_{1}}}$,

$T_{2}^{2}=p T_{3}^{3}=p$

$T_{4}^{4}=-(p+\rho) \cosh ^{2} \alpha+p-2 q_{1} \frac{\sinh \alpha}{t \xi_{1}}$

With the help of equations (2) - (6), the field equations (1) reduces to

$$
\begin{aligned}
& -\left[\xi_{1}(S-1)-\frac{1}{2}\left(S^{2}-2 S+Q\right)\right] t^{-2}-\frac{\omega}{2} \varphi^{n} \varphi_{4}{ }^{2} \\
& =(p+\rho) \sinh ^{2} \alpha+p+2 q_{1} \frac{\sinh \alpha}{t^{\xi_{1}}}, \\
& -\left[\xi_{2}(S-1)-\frac{1}{2}\left(S^{2}-2 S+Q\right)\right] t^{-2}- \\
& \frac{\omega}{2} \varphi^{n} \varphi_{4}{ }^{2}=p \\
& -\left[\xi_{3}(S-1)-\frac{1}{2}\left(S^{2}-2 S+Q\right)\right] t^{-2}-\frac{\omega}{2} \varphi^{n} \varphi_{4}{ }^{2} \\
& =p, \\
& \frac{1}{2}\left(Q-S^{2}\right) t^{-2}-\frac{\omega}{2} \varphi^{n} \varphi_{4}{ }^{2} \\
& =(p+\rho) \cosh ^{2} \alpha-p+2 q_{1} \frac{\sinh ^{2}}{t^{\xi}}, \\
& (p+\rho) \sinh \alpha \cosh \alpha t^{\xi_{1}}+q_{1} \cosh \alpha+q_{1} \frac{\sinh ^{2} \alpha}{\cosh \alpha} \\
& =0
\end{aligned}
$$

Where suffix 4 after field variable denotes ordinary differentiation with respect to cosmic time $t$ and

$$
S=\sum_{i=1}^{3} \xi_{i} \quad \& \quad Q=\sum_{i=1}^{3} \xi_{i}{ }^{2}
$$


For Kasner form of Bianchi type I model, the average scale factor $R$ and the spatial volume $V$ are given by

$$
\begin{aligned}
& R(t)=\left(t^{2 \xi_{1}+2 \xi_{2}+2 \xi_{3}}\right)^{\frac{1}{3}} \\
& V=R^{3}=t^{2 \xi_{1}+2 \xi_{2}+2 \xi_{3}}
\end{aligned}
$$

The directional mean Hubble's parameter for this model is given by

$$
\begin{aligned}
H & =\frac{1}{3}\left(H_{x}+H_{y}+H_{z}\right) \\
& =\frac{2}{3}\left(\frac{\xi_{1}}{t}+\frac{\xi_{2}}{t}+\frac{\xi_{3}}{t}\right)
\end{aligned}
$$

Where $H_{x}, H_{y}$ and $H_{z}$ are the directional Hubble's parameters in the directions of $\mathrm{x}, \mathrm{y}$ and $\mathrm{z}$ axes respectively which are given by

$$
H_{x}=\frac{2 \xi_{1}}{t}, H_{y}=\frac{2 \xi_{2}}{t}, \quad H_{z}=\frac{2 \xi_{3}}{t}
$$

The anisotropic expansion parameter $A_{m}$ for the universe is defined as

$A_{m}=\frac{1}{3} \sum_{i=1}^{3}\left(\frac{\Delta H_{i}}{H}\right)^{2}=\frac{9 Q}{S^{2}}$,

where $\Delta H_{i}=H_{i}-H$

This anisotropic parameter is one of the important candidate which describes the expansion history of the universe. The universe expands isotropically if $A_{m}=0$ and the universe expands anisotropically for non-vanishing value of anisotropic parameter.

The deceleration parameter,

$$
q=-\frac{R R_{44}}{R_{4}{ }^{2}}=\frac{3}{2\left(\xi_{1}+\xi_{2}+\xi_{3}\right)}-1
$$

Also the expansion scalar $\Theta$ and the shear scalar $\sigma$ are given by

$$
\begin{gathered}
\Theta=3 H=2\left(\frac{\xi_{1}}{t}+\frac{\xi_{2}}{t}+\frac{\xi_{3}}{t}\right) \\
\sigma^{2}=\frac{1}{12}\left[\left(\frac{g_{11,4}}{g_{11}}-\frac{g_{22,4}}{g_{22}}\right)^{2}+\left(\frac{g_{22,4}}{g_{22}}-\frac{g_{33,4}}{g_{33}}\right)^{2}\right. \\
\left.+\left(\frac{g_{33,4}}{g_{33}}-\frac{g_{11,4}}{g_{11}}\right)^{2}\right]
\end{gathered}
$$

$$
\begin{aligned}
& \cosh \propto=1 \\
& \sinh \propto=0
\end{aligned}
$$

$$
\begin{array}{cc}
(p+\rho) \sinh \alpha \cosh \alpha & t^{\xi_{1}}+q_{1} \cosh \alpha \\
=0 & +q_{1} \frac{\sinh ^{2} \alpha}{\cosh \alpha} \\
\varphi_{44}+\frac{\varphi_{4}}{t}+\frac{n}{2} \frac{\varphi_{4}{ }^{2}}{\varphi}=0
\end{array}
$$

\section{SOME PHYSICAL PARAMETERS OF THE MODEL FOR $S=1$}

Using equations (3), (23) and (27), heat conduction vector $\mathrm{q}_{1}, \mathrm{q}_{4}$; pressure $\mathrm{p}$, density $\rho$ and tilt angle $\propto$ becomes

$\mathrm{q}_{1}=\frac{-(\mathrm{p}+\rho) \sinh \propto \cosh ^{2} \propto \mathrm{t}^{\xi_{1}}}{\cosh 2 \propto}$

$\mathrm{p}=\rho=\frac{1}{2}(\mathrm{Q}-1) \mathrm{t}^{-2}-\frac{\omega}{2} \mathrm{C}_{1} \mathrm{t}^{-2}$

$p=\rho$ i.e., a stiff fluid; velocity of sound is equal to the velocity of light. Hence given metric satisfy Zel'dovich equation of state. According to Brevik et.al. [27-28], Kasner metric with viscous fluid in Einstein theory is not anisotropic and that of Kasner metric with non-viscous fluid satisfying Zel'dovich equation of state is to be anisotropic.

Also from equations (23), (25), (28) and (29),

Tilted angles $\cosh \propto, \sinh \propto$ are independent of cosmic time.

Using equations (30), (31); heat conduction vector $\mathrm{q}_{1}, \mathrm{q}_{4}$ and fluid flow vectors becomes $\mathrm{u}^{1}, \mathrm{u}^{4}$ 


$$
\begin{array}{lll}
\mathrm{q}_{1}=0 & \& & \mathrm{q}_{4}=0 \\
\mathrm{u}^{1}=0 & \& & \mathrm{u}^{4}=1
\end{array}
$$

From equations (8) $-(10)$ along with $\sinh \propto=0$ and $\mathrm{S}=1$ gives

$$
\xi_{1}=\xi_{2}=\xi_{3}=\frac{1}{3}
$$

The average scale factor $R$ and the spatial volume $V$ becomes

$$
\begin{aligned}
& R(t)=t^{\frac{2}{3}} \\
& V=R^{3}=t^{2}
\end{aligned}
$$

The directional mean Hubble's parameter for this model is given by

$$
H=\frac{2}{3} \frac{1}{t}
$$

\section{RESULTS AND DISCUSSION}

We plot the graphs of spatial volume and Hubble parameter with respect to cosmic time.

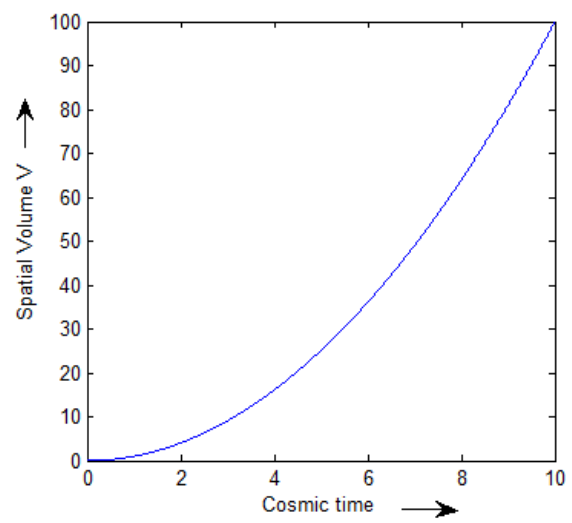

Fig:1 Plot of Spatial Volume versus Cosmic time

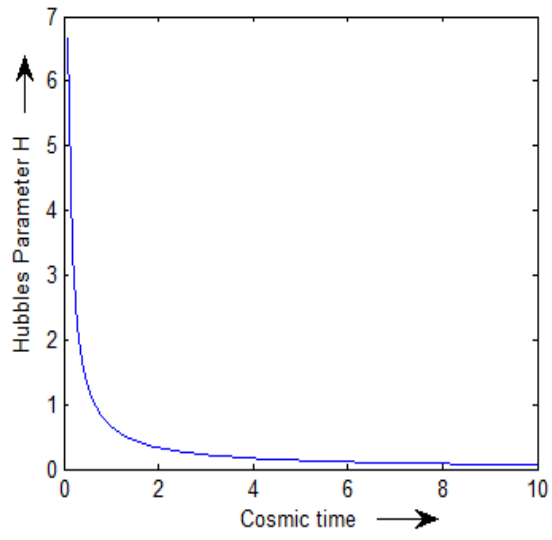

Fig:2 Plot of Hubble's Parameter versus Cosmic time.
From above figures, it is observed that the spatial volume is directly proportional to time. It increases with increase in time. Increase in cosmic time results into drop in Hubble's parameter. As $t \rightarrow \infty$, the tilted universe approaches to infinite volume and Hubble's parameter becomes zero.

The anisotropic expansion parameter $A_{m}$,

$$
A_{m}=9 Q
$$

For the tilted Kasner cosmological model this anisotropic parameter is non-zero since $A_{m} \neq 0$. Hence the universe expands anisotropically.

The deceleration parameter,

$$
q=\frac{1}{2}
$$

Here $q>0$, hence the tilted universe is decelerating for Kasner metric in Saez-Ballester theory. This observation is inconsistent with the present day observation.

Also the expansion scalar $\Theta$ and the shear scalar $\sigma$ are given

$$
\begin{gathered}
\Theta=\frac{2}{t} \\
\sigma^{2}=\frac{2}{3 \mathrm{t}^{2}}\left(\mathrm{Q}-\frac{1}{3}\right)
\end{gathered}
$$

The Hubble parameter, expansion scalar and shear scalar decreases with increase in time. And they will be zero as $t \rightarrow \infty$.It results into expansion of the tilted universe. Since $\lim _{t \rightarrow \infty} \frac{\sigma}{\Theta} \neq 0$, the model does not approach to isotropy.

\section{CONCLUSION}

We studied the tilted Bianchi type-I cosmological model of Kasner form with non viscous fluid in SaezBallester theory of gravitation. To obtain physical parameters of the model, we used the condition $S=\xi_{1}+$ $\xi_{2}+\xi_{3}=1$. It is observed that the metric (1) with nonviscous fluid satisfy Zel'dovich equation of state, also anisotropic parameter $A_{m} \neq 0$, hence the tilted Kasner universe expands anisotropically. The deceleration parameter $q>0$, hence the universe is decelerating.

As time rises infinitely, the tilted universe approaches to infinitely large volume. The Hubble parameter, expansion scalar and shear scalar drops with rise in cosmic time, will be zero as $t \rightarrow \infty$. It results into expansion of the tilted universe. Also the energy density and pressure are zero when $t \rightarrow \infty$. Since $\lim _{t \rightarrow \infty} \frac{\sigma}{\Theta} \neq 0$, the model does not approach to isotropy. Tilted angle $\cosh \alpha, \sinh \alpha$ are constant and they are independent of cosmic time. 


\section{REFERENCES}

[1] King, R.; Ellis, G. G. R. :Comm. Math. Phys.(1973), 31, 209.

[2] Ellis, G. G. R.; King, A. R. :Comm. Math. Phys. (1974), 38, 119.

[3] Collins, C. B.; Ellis, G. G. R.: Phys. Rep. (1979), 56, 65.

[4] Matravers, D. R.; Madsen, M. S.; Vogel D. L.: (1958). Astrophys. Space Sci., 112, 193.

[5] Dunn, K. A. ;Tupper, B. O. J.: Astrophys. (1980) J., 235, 307

[6] Horwood, J. T.; Hancock, M. J.;Wainwright : Preprint gr$\operatorname{ac}(2002) / 0210031$.

[7] Hewitt, G. G.; Horwood, J. T.; Wainstright, J. : Preprint grac(2002)/0211071.

[8] Apostolopoulos, P. S.: Preprint gr-ac(2003)/0310033.

[9] Bali, R.; Sharma, K.: Pramana.J. (2002), 58,457.

[10] Pradhan, A.; Rai, A. :Astophys. J. (2003), 286, 347.

[11] Pawar, D. D.; Bhaware, S. W.; A. G. Deshmukh : Rom. J. Phys. (2009), 54, 187-194.

[12] Bali, R.; Meena, B.L.: Astrophys. J. (2002) 281565.

[13] Pawar, D. D.; Dagwal, V. J.: Bulg. J. Phys. (2010), 37, 165-175.

[14] Sahu., S. K.: J. Mod. Phys. (2010), 1, 67-69.

[15] Sahu, S.K. et al.: Int. J. Theor. Phys.,(2016)55, 526-534.

[16] Pawar, D. D.; Dagwal, V. J.:International Journal of Mathematical Archive. (2014), 5, 9,113-118.

[17] Pawar, D. D.; Dagwal, V. J.:Prespacetime Journal (2015), 6,11, 1165-117

[18] Saez, D.; Ballester, V. J.: Physics Letters A (1986),Vol. 133, (1986), p. 477.

[19] Shri Ram, Tiwari, S.K.: Astrophys. and Space Sci.(1998), 259, 91.

[20] Singh, C.P., Shri Ram: Astrophys. and Space Sci.(2003), 284,1199.

[21] Katore , S.D. : Prespacetime Journal (2011) 2 (4).

[22] Shivdas D. Katore, A. Y. Shaikh, G. B. Tayade: Prespace time (2012), Vol 3, No.7.

[23] Mishra, B., Sahoo, P.K.: Astrophys. and Space Sci.(2014), 349, 491-499.

[24] Pawar, D.D.; Panpatte, M. K.: Prespacetime Journal (2016), Vol 7, No 8

[25] Pawar, D.D., Solanke, Y.S.: arXiv/abs/1602.05222v1, (gr-qc), (2016).

[26] Verma, M.K., Chandel, S. \& Ram, S.: Pramana - J Phys(2017) 88: 8. doi:10.1007/s12043-016-1317-4

[27] Brevik, I. ; Pettersen, S.V. : Phys. Rev. D 61 (2000)127305.

[28] Brevik, I. ; Pettersen, S.V. : Phys. Rev. D 56 (1997) 3322.

[29] Hawking, S.W.; Ellis, G.F.R.: The large scale structure of spacetime (1980) (Cambridge Monographs on Mathematical Physics).

[30] Cataldo , M.; Sergio del Campo: Phys. Rev. D 61 (2000) 128301.

[31] Cataldo, M., Cruz, N., del Campo, S., Lepe, S.: Phys. Lett. (2001) B 509, 138.

[32] Halpern, P.:Phys. Rev. D (2001)63, 024009.

[33] Cataldo, M.; del Campo; S., Salgado, P.: Phys. Rev. (2001) D 63, 063503.

[34] Lee, S.: gr-qc (2008) /0811 